\title{
In situ measurement of shrinkage and temperature profile in microwave- and conventionally-sintered hydroxyapatite bioceramic
}

Muralithran G. Kutty ${ }^{1,2}$, Sarit B. Bhaduri ${ }^{3}$, Huan Zhou ${ }^{3,4}$ and Alireza Yaghoubi ${ }^{5 *}$

${ }^{1}$ Department of Restorative Dentistry, Faculty of Dentistry, University of Malaya, Kuala Lumpur 50603, Malaysia

${ }^{2}$ Department of Materials and Metallurgical Engineering, University of Idaho, Moscow, ID 83844, USA

${ }^{3}$ Multi-functional Materials Laboratory, University of Toledo, Toledo, OH 43606, USA

${ }^{4}$ Institute of Biomedical Engineering and Health Sciences, Changzhou University, Changzhou, Jiangsu 213164, China

${ }^{5}$ Center for High Impact Research, University of Malaya, Kuala Lumpur 50603, Malaysia

*Correspondence to: yaghoubi@siswa.um.edu.my

Tel.: +60173470750

Fax: +60379677727

\section{Abstract}

The clear advantages of microwave (MW) sintering in terms of energy savings as well as superior mechanical properties have led to the popularity of this technique in recent years, especially in the bioceramics community. However to date, no study has established a systematic comparison between the sintering behavior of the most commonly used bioceramic, hydroxyapatite (HA), during MW and conventional sintering. Here, we utilize in situ dilatometry along with optical pyrometry to monitor the rate of shrinkage and temperature profile for various sintering conditions of HA. Temperature-dependent dielectric measurements indicate that microwave coupling occurs around $900^{\circ} \mathrm{C}$ and the maximum linear shrinkage is reached, on average, 4 times faster in MW sintering. Finally we show that by tuning the MW power, one can effectively benefit from the non-linear increase in relative permittivity to exert control over the evolution of microstructures and hence achieve the desired combination of physical properties for personalized biomedical applications.

Keywords: Biomaterials; Metallurgy; Dielectrics; Sintering; Thermal analysis; Dilatometry

\section{Introduction}

The dominant mode of heat transfer in primary stages of conventional sintering is convective and therefore temperature gradient in the sample can widely vary before the surface is sufficiently heated and thermal conduction takes over. In microwave (MW) sintering on the contrary, dielectric heating gives rise to a nearly uniform 
volumetric heat transfer as the microwaves penetrate and dissipate throughout the bulk concurrently. This major difference leads to a number of significant benefits including higher electricity-to-heat conversion efficiency, faster heating rate, lower energy consumption and remarkably less heat leakage. Some studies have suggested that MW sintering can achieve up to $90 \%$ better energy efficiency as compared to conventional electric furnaces [1].

Beside the lower energy consumption and a shorter processing time, both of which contribute positively to the overall cost savings, MW-sintered samples often show improved mechanical properties. This can be attributed to the lower thermal stress in the bulk due to uniform volumetric heating as well as the faster heating rate that leads to smaller grain size [2]. A finer microstructure does not only contribute towards better mechanical properties, but also has been shown to have biological advantages during in vitro studies. Bose et al. [3] have reported that along with improved compressive strength, fracture toughness and hardness, MW-sintered hydroxyapatite (HA) samples with smaller grain size in the nanoscale regime induce a more prominent expression of vinculin proteins which are responsible for attachment of bone cells to the surface. MTT assays also indicated improved cell proliferation on finer grains as opposed to coarse-grained samples. In the particular case of biomaterials, MW sintering may also enable further customization of the porosity for increased bioactivity through optimization of sintering parameter [4] or the starting material's morphology [5]. More sophisticated MW-sintered structures for biomedical implants with functionally-graded porosity and distinct surface topographies that nearly double cell viability and therefore accelerate bone healing in early stages have also been recently demonstrated [6]. However more than two decades after Fang and colleagues [7] reported the first MW sintering of HA bioceramics with enhanced processing efficiency and mechanical properties, despite all the advantages, a systematic study of the sintering behavior of HA in MW versus conventional sintering is still lacking. Although in recent years, new bioceramics such as calcium sulfates [8] as well as silicates [9] including magnesium silicates [10,11] and calcium silicate [12] have been explored as more mechanically superior successors of calcium phosphates, HA, as the main natural biomineral of bone remains the most relevant bioceramic [13]. In this work, we use dilatometry and pyrometry techniques to closely study the real-time sintering behavior of HA under various conditions. High-temperature dielectric measurements based on cavity perturbation methods suggest that HA-MW coupling takes place at about $900{ }^{\circ} \mathrm{C}$. In situ measurements of linear shrinkage clearly show the notably faster rate of densification which on average is 4 times that of conventional sintering at the same holding temperature. Lastly, we discuss the effect of MW power during sintering on microstructural evolution and variation in mechanical properties of HA. 


\section{Material and methods}

\subsection{Materials preparation and sintering}

A detailed description of synthesis of nanocrystalline HA via precipitation can be found elsewhere [14]. The powder was sieved (mesh $<45 \mu \mathrm{m}$ ) and pressed into discs $20 \mathrm{~mm}$ in diameter by uniaxial pressing at $40 \mathrm{MPa}$ and subsequently by cold isostatic pressing (CIP) at $150 \mathrm{MPa}$ for 20 minutes. The HA green compacts were then sandwiched between two $\mathrm{SiC}$ suceptors and surrounded with alumina fiber insulation prior to sintering. Sintering was carried out in an industrial MW furnace (M.M.T., Knoxville, TN) equipped with a variable power output magnetron source capable of operating up to $3 \mathrm{~kW}$ at $2.45 \mathrm{GHz}$. The cavity is rather large and over-moded and therefore ensures proper mixing of MW modes and a homogeneously distributed field. The total sintering time was kept constant at 20 minutes and the MW power input was continuously monitored and tuned to maintain the sintering temperature. The input power therefore slightly fluctuated during sintering and the values presented throughout this report merely represent the average figure.

Conventional sintering was carried out with a heating rate of $25^{\circ} \mathrm{C} / \mathrm{min}$ and a holding time of 10 minutes.

\subsection{In situ measurements}

In order to record in situ data on shrinkage, a push rod dilatometer (Netzsch $402 \mathrm{ES}$ ) of horizontal design was attached to the MW furnace. The measuring rod was inserted through a port on the microwave door and sealed with aluminum mesh (see Fig. S1). To construct the real-time temperature profile, the use of the thermocouple within the dilatometer unit was not feasible due to MW coupling and therefore a two-color pyrometer (Mikron L77, Mikron Inc. Oakland, NJ) was utilized instead. Temperature data points were obtained with a data acquisition system. The pyrometer was focused on the sample surface in-between the susceptors through a hole created along the insulation material. The shrinkage data from the dilatometer and pyrometer were synchronized to obtain the accurate profile plot. High-temperature dielectric properties were measured based on a variation of cavity perturbation method using the approach established by Tinga and colleagues [15]. Dielectric constant $\left(\varepsilon^{\prime}\right)$ and loss factor $\left(\varepsilon^{\prime \prime}\right)$ were calculated for each temperature interval using the following equations:

$$
\begin{gathered}
\varepsilon^{\prime}=1+0.539\left(\frac{\mathrm{V}_{\mathrm{o}}}{\mathrm{V}_{\mathrm{s}}}\right)(\Delta \mathrm{f}) \\
\varepsilon^{\prime \prime}=0.269\left(\frac{\mathrm{V}_{\mathrm{o}}}{\mathrm{V}_{\mathrm{s}}}\right)\left(\frac{1}{\mathrm{Q}_{\mathrm{s}}}-\frac{1}{\mathrm{Q}_{\mathrm{o}}}\right)
\end{gathered}
$$


wherein $V_{S}$ and $V_{O}$ are the volume of the sample and the cavity, respectively, $Q_{O}$ and $Q_{S}$ are the quality factors of the empty and sample loaded cavity respectively, and

$$
\Delta \mathrm{f}=\frac{\mathrm{f}_{\mathrm{o}}-\mathrm{f}_{\mathrm{s}}}{\mathrm{f}_{\mathrm{s}}}
$$

in which $f_{O}$ and $f_{S}$ are resonant frequency of the empty and sample-loaded cavity, respectively [16].

The effect of the $\mathrm{SiC}$ susceptor was taken into consideration by repeating the measurements with and without the HA sample. It should also be noted that dielectric measurements were obtained on a dedicated setup to avoid any interference from the dilatometer.

\subsection{Physical and mechanical properties}

Average grain size was determined using the intercept method based on ASTM E112-96. Relative density was measured in accordance with ASTM B962 standard.

Vickers indentation tests were done on polished samples to obtain hardness and fracture toughness. A microindenter was used with a load of 500g applied for 15 seconds. In total, 10 indents were made for each sample and they were distributed all around the surface. Fracture toughness $\left(\mathrm{K}_{1 \mathrm{c}}\right)$ was estimated from the following equation:

$$
\mathrm{K}_{1 \mathrm{c}}=\mathrm{A}(\mathrm{E} / \mathrm{H})^{1 / 2} \mathrm{Pc}^{-3 / 2}
$$

where A equals 0.016 . $\mathrm{E}$ is the elastic modulus, $\mathrm{H}$ is the hardness, $\mathrm{c}$ is half the indentation crack length, and $\mathrm{P}$ is the applied load. In these calculations, E was taken as $115 \mathrm{GPa}[17]$.

\section{Results \& Discussion}

A number of researchers have studied the dielectric properties of HA sintered under different conditions. Valdes et $a l$. for instance have reported frequency-dependent measurements that show capacitive or resistive response for slowly cooled and quenched samples, respectively [18]. Temperature-dependent dielectric measurements have also been performed, although either at relatively low temperatures or without looking at the densification process. Ikoma and colleagues [19] have described the dielectric loss mechanism of HA up to $700 \mathrm{~K}$ in terms of reorientation of hydroxyl ions and defects during formation of oxyapatite while the more recent paper by Gittings et al. [20] aimed at examining the concept of implant electrical stimulation by studying the effect of dehydration of HA on impedance properties from $200{ }^{\circ} \mathrm{C}$ to $1000{ }^{\circ} \mathrm{C}$. Our aim here on the other hand is to understand the mechanism of dielectric heating using high-temperature dielectric loss measurements at the onset of MW coupling of HA. According to our measurements (Fig. 1), the dielectric constant and dielectric loss factor in HA sample do not show 
a significant elevation until around $900{ }^{\circ} \mathrm{C}$. This is the temperature at which both dielectric measurements undergo a nearly threefold increase as a clear indication of the effective HA-MW coupling. We need to put emphasis on the word "effective" given that even at lower temperatures, there is MW dissipation (and hence dielectric heating), however due to the lack of uniform volumetric heating, the rate at which it takes place is essentially different. Previous measurements of loss tangent at lower temperatures $\left(<500^{\circ} \mathrm{C}\right)$ agree with this interpretation [21]. This trend in MW coupling was also taken into account as part of the real-time pyrometry of HA samples during sintering. It is clear from Fig. 2 that regardless of the holding temperature, the profile does not undergo any noticeable change at about $900{ }^{\circ} \mathrm{C}$ for a few minutes before rapidly increasing. Another interesting observation that is in agreement with previous findings about the temperature-dependent increase of relative permittivity in HA [19] is the non-linear relationship between the shrinkage rate and the holding temperature (not to be confused with the term "linear shrinkage rate" defined as $\left.\mathrm{dL} / \mathrm{L}_{0}\right)$. The linear shrinkage rates corresponding to sintering at $1 \mathrm{~kW}(1000$ $\left.{ }^{\circ} \mathrm{C}\right)$ and $1.2 \mathrm{~kW}\left(1100{ }^{\circ} \mathrm{C}\right)$ show a gradual increase while the ones corresponding to sintering at $1.5 \mathrm{~kW}\left(1200{ }^{\circ} \mathrm{C}\right)$ and $1.8 \mathrm{~kW}\left(1300{ }^{\circ} \mathrm{C}\right)$ exhibit very steep shrinkage rates. Although one must consider that for the first few minutes, as the sample is reaching the holding temperature, the heating rate significantly varies with MW power and indeed this would have the most dominant effect on the increasing shrinkage rate that we observe here. Only in the subsequent stage when the maximum temperature is already achieved, the idea of temperature-dependent increase in relative permittivity becomes relevant. In comparison, in conventional sintering using a standard tube furnace (Fig. 3) due to the constant heating rate, shrinkage is perfectly linear. A fact that is further compatible with the nature of conductive heating. This complex interaction between microwaves and the sample during sintering certainly influences the densification process. Since the runs were limited to 20 minutes, the sample sintered at $1 \mathrm{~kW}$ only reached a shrinkage of $15.3 \%$. On the other hand, a maximum shrinkage of $24.0 \%$ was achieved in the $1.5 \mathrm{~kW}$ run in a duration of 14 minutes, whereas the same figure was achieved in the $1.8 \mathrm{~kW}$ run in a considerably shorter duration of 12 minutes. In the conventional sintering on the contrary, the holding time was set at 60 minutes due to the limitation of the heating rate of the furnace to reach the set temperatures. The results (Fig. 3) indicate that the maximum shrinkage achieved was $25.0 \%$ when sintered at $1300{ }^{\circ} \mathrm{C}$ with a runtime of 60 minutes. A comparison of the two methods clearly suggests that microwave sintering achieved equivalent densities at a shorter duration. For example, when we compare the data of the samples sintered at $1200{ }^{\circ} \mathrm{C}$, a shrinkage of $24 \%$ was achieved for both sintering techniques but with the MW sintering taking on average one fourth of the time. Moreover, despite the 
faster heating rate, the samples sintered by MW also showed less cracks due to the uniform volumetric heating. To have a better visual understanding of the heating as well as the shrinkage rate in both methods, we encourage the readers to see the side-by-side curves of Fig. S2 in the supplementary file.

With regard to the overall physical attributes, as shown in Table 1, we further introduced 3 intermediate data points beside the primary 4 samples for shrinkage and temperature profile measurements. This was intended as to more clearly represent the earlier mentioned non-linear permittivity-temperature relationship as well as the greater potential for customization of properties. For example, porous samples can be rapidly prepared at low powers. The relative density of the sample sintered for a duration of 20 minutes at $1 \mathrm{~kW}$ is $90.8 \%$ as compared to the highest value of $98 \%$ for the samples sintered at $1.8 \mathrm{~kW}$. In terms of the evolution of microstructures, the sub-micron grains are dominant in samples sintered at $1 \mathrm{~kW}$ and $1.1 \mathrm{~kW}$ with grain sizes of $0.28 \pm 0.12 \mu \mathrm{m}$ and $0.35 \pm 0.12 \mu \mathrm{m}$, respectively. At higher powers in general, there is a rapid increase in grain size. However there are several anomalies that can prove advantageous in tuning the properties for specific applications; for instance the sample sintered at $1250^{\circ} \mathrm{C}(\sim 1.65 \mathrm{~kW})$ has the highest average grain size of $2.21 \pm 0.74 \mu \mathrm{m}$ as opposed to only $1.24 \pm 0.45$ at $1.8 \mathrm{~kW}$. Similarly at $1.35 \mathrm{~kW}$ and $1.5 \mathrm{~kW}$, the relative density is about the same while the average grain size is twice larger for the latter $(0.77 \pm 0.52 \mu \mathrm{m}$ versus $1.40 \pm 0.39 \mu \mathrm{m})$. These anomalies are also observed in the mechanical properties. For the Vickers hardness, the sample sintered at $1 \mathrm{~kW}$ and $1.35 \mathrm{~kW}$ have the highest and lowest values of $3.85 \pm 0.42 \mathrm{GPa}$ and $6.38 \pm 0.10 \mathrm{GPa}$, respectively. The fracture toughness in contrast shows an inverse relationship with hardness measuring highest at $1.75 \pm 0.17 \mathrm{MPa} \sqrt{\mathrm{m}}$ for $1 \mathrm{~kW}$ and lowest at $1.16 \pm 0.10$ $\mathrm{MPa} \sqrt{\mathrm{m}}$ for $1.35 \mathrm{~kW}$.

It must be emphasized that to some extent, the anomalies can be attributed to the relatively large standard deviation in grain size which despite the uniform volumetric heating may be an outcome of increased heat dissipation from the surface that in turn leads to uneven evolution of microstructures (for example see the scanning electron micrograph of Fig. S3 showing the polished surface of the sample sintered at $1200^{\circ} \mathrm{C}$ ). Hence, the mechanical behaviors in principle can large vary depending on the indentation size and where the it actually takes place. Fig. S4 shows the difference in readings from nano-indentation and micro-indentation. It is clear that the former shows a more consistent trend with the average grain size, although sudden changes are still present and can be highly temperature-dependent at certain intervals (for example, the peak in grain size at $1250^{\circ} \mathrm{C}$ ). 


\section{Conclusions}

In summary, we have utilized in situ measurement techniques to study the high-temperature dielectric properties, temperature profile and shrinkage rate of HA bioceramic in real-time for the first time. HA is found to couple with MWs at temperatures about $900{ }^{\circ} \mathrm{C}$ beyond which the dielectric loss and hence heating is significantly increased. Synchronized dilatometry and pyrometry suggests non-linear relationship between shrinkage rate and temperature which can be attributed to the temperature-dependent relative permittivity of HA. This property is advantageous and enables the fabrication of biomedical implants with customized properties on average 4 times faster than conventional sintering with considerably less power consumption and improved mechanical properties due to the rapid heating rate.

\section{Acknowledgements}

This study was funded by NSF under Grant No. DMI-0085100. A.Y. and M.G.K. acknowledge support from University of Malaya under HIR Grant UM.C/625/1/HIR/MOHE/CHAN/09 and UMRG grant RG538-13HTM, respectively. The authors would like to thank W.R. Tinga for his contributions to dielectric measurements.

\section{References}

[1] Katz JD. Annu Rev Mater Sci 1992;22:153-170.

[2] Oghbaei M, Mirzaee O. J Alloy Compd 2010;494:175-189.

[3] Bose S, Dasgupta S, Tarafder S, Bandyopadhyay A. Acta Biomater 2010;6:3782-3790.

[4] Wang X, Fan H, Xiao Y, Zhang X. Mater Lett 2006;60:455-458.

[5] Fang Y, Agrawal DK, Roy DM, Roy R. J Mater Res 1992;7:490-494.

[6] Kutty MG, De A, Bhaduri SB, Yaghoubi A. ACS Appl Mater Interfaces 2014;6:13587-13593.

[7] Fang Y, Agrawal DK, Roy DM, Roy R. J Mater Res1994;9:180-187.

[8] Huan Z, Chang J. Acta Biomater 2007;3:952-960.

[9] Diba M, Goudouri OM, Tapia F, Boccaccini, AR. Curr Opin Solid State Mater Sci 2014;18:147-167.

[10] Afshar-Mohajer M, Yaghoubi A, Ramesh S, Bushroa AR, Chin KMC, Tin CC, Chiu WS. Appl Surf Sci 2014;307:1-6.

[11] Ramesh S, Yaghoubi A, Lee KS, Chin KC, Purbolaksono J, Hamdi M, Hassan MA. J Mech Behav Biomed Mater 2013;25:63-69.

[12] Ni S, Chang J, Chou L, Zhai W. J Biomed Mater Res Part B Appl Biomater 2007;80:174-183.

[13] Sadat-Shojai M, Khorasani MT, Dinpanah-Khoshdargi E, Jamshidi A. Acta Biomater 2013;9:7591-7621. 
[14] Tan CY, Yaghoubi A, Ramesh S, Adzila S, Purbolaksono J, Hassan MA, Kutty MG. Ceram Int 2013;39:89798983.

[15] Tinga W, Xi W. J Microw Power Electromagn Energy 1993;28:93-103.

[16] Liao X, Raghavan VGS, Meda V, Yaylayan V. J Microw Power Electromagn Energy 2001;36:131-138.

[17] Wang, J, Shaw LL. Biomaterials 2009;30,:6565-6572.

[18] Prieto Valdes JJ, Victorero Rodriguez A, Guevara Carrio J. J Mater Res 1995;10:2174-2177.

[19] Ikoma T, Yamazaki A, Nakamura S, Akao M. J Mater Sci Lett 1999;18:1225-1228.

[20] Gittings JP, Bowen CR, Dent AC, Turner IG, Baxter FR, Chaudhuri JB. Acta Biomater 2009;5:743-754.

[21] Zakharov NA, Orlovskii VP. Tech Phys Lett 2001;27:629-631.

\section{Figure captions}

Figure 1: Variation of hydroxyapatite's dielectric constant $\left(\varepsilon^{\prime}\right)$ and dielectric loss factor $\left(\varepsilon^{\prime \prime}\right)$ with temperature at 915 $\mathrm{MHz}$.

Fig. 2. In situ temperature profile (after MW coupling, T $900^{\circ} \mathrm{C}$ ) and linear shrinkage for MW-sintered HA. See

Table 1 for details of sample A-D.

Fig. 3. In situ temperature profile and linear shrinkage for conventionally sintered HA at different temperatures: A = $1000^{\circ} \mathrm{C}, \mathrm{B}=1100^{\circ} \mathrm{C}, \mathrm{C}=1200{ }^{\circ} \mathrm{C}$ and $\mathrm{D}=1300^{\circ} \mathrm{C}$.

\section{Tables}

Table 1: Change of physical and mechanical properties with MW power (SD: standard deviation). 3 intermediate points are added to emphasize the anomalies.

\begin{tabular}{|c|c|c|c|c|c|c|}
\hline Sample & $\begin{array}{l}\text { Average } \\
\text { MW } \\
\text { Power } \\
\text { (kW) }\end{array}$ & $\begin{array}{l}\text { Temp. } \\
\left({ }^{\circ} \mathrm{C}\right)\end{array}$ & $\begin{array}{l}\text { Relative } \\
\text { Density } \\
\text { (\%) }\end{array}$ & $\begin{array}{l}\text { Grain Size } \\
\pm \mathrm{SD}(\mu \mathrm{m})\end{array}$ & $\underset{(\mathrm{GPa})}{\mathrm{Hv}} \pm$ SD & $\begin{array}{l}\text { K1c } \pm \text { SD } \\
(\mathrm{MPa} \sqrt{\mathrm{m})}\end{array}$ \\
\hline $\mathbf{A}$ & 1.00 & 1000 & 90.8 & $0.28 \pm 0.12$ & $3.85 \pm 0.42$ & $1.75 \pm 0.17$ \\
\hline - & 1.10 & 1050 & 95.2 & $0.35 \pm 0.12$ & $5.96 \pm 0.89$ & $1.72 \pm 0.24$ \\
\hline B & 1.20 & 1100 & 96.5 & $1.32 \pm 0.34$ & $6.19 \pm 0.41$ & $1.33 \pm 0.10$ \\
\hline - & 1.35 & 1150 & 96.7 & $0.77 \pm 0.52$ & $6.38 \pm 0.3$ & $1.16 \pm 0.10$ \\
\hline $\mathbf{C}$ & 1.50 & 1200 & 96.6 & $1.40 \pm 0.39$ & $6.13 \pm 0.27$ & $1.45 \pm 0.15$ \\
\hline- & 1.65 & 1250 & 97.0 & $2.21 \pm 0.74$ & $5.39 \pm 0.38$ & $1.18 \pm 0.06$ \\
\hline D & 1.80 & 1300 & 98.0 & $1.24 \pm 0.45$ & $6.00 \pm 0.43$ & $1.38 \pm 0.14$ \\
\hline
\end{tabular}




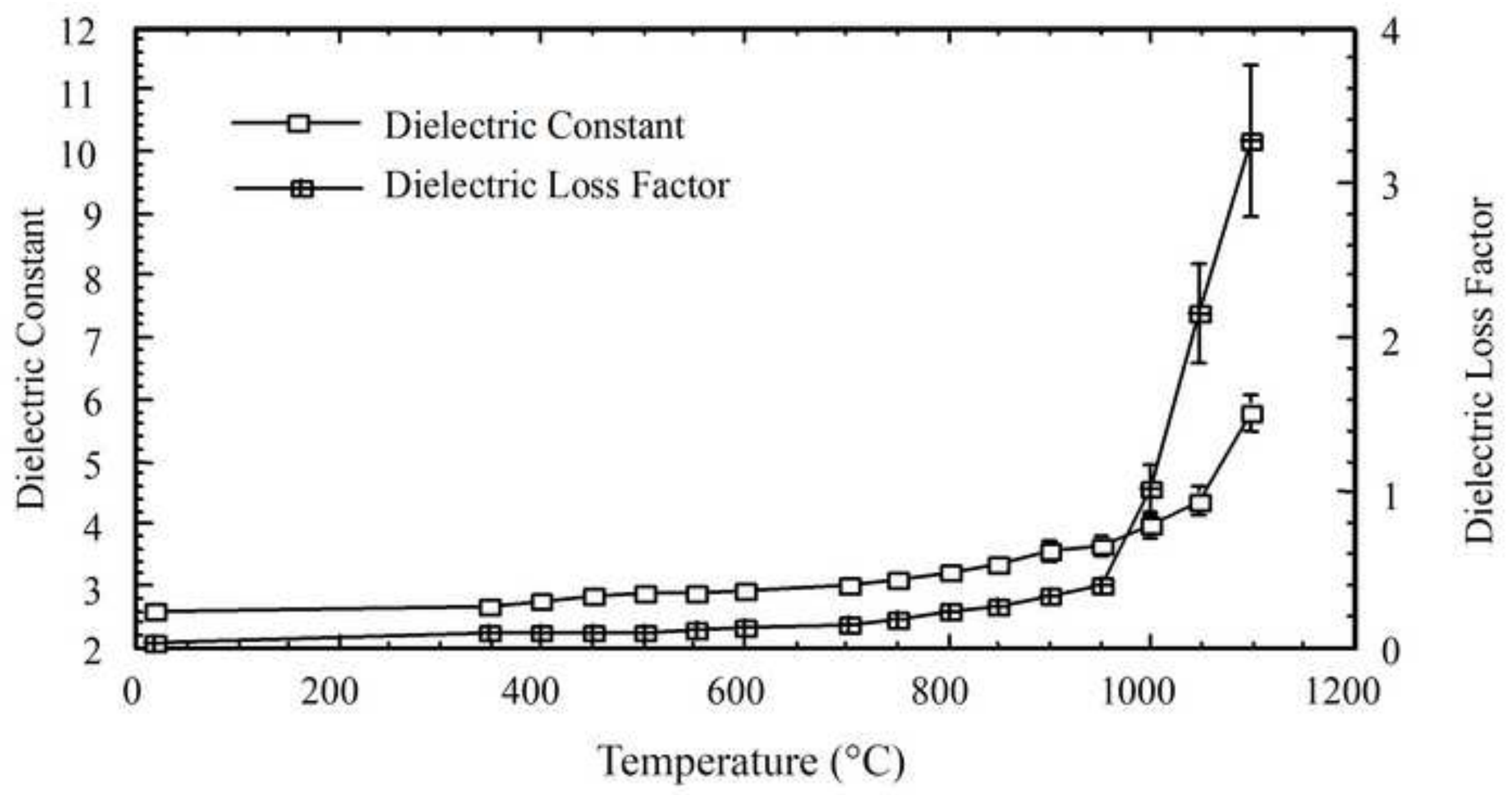




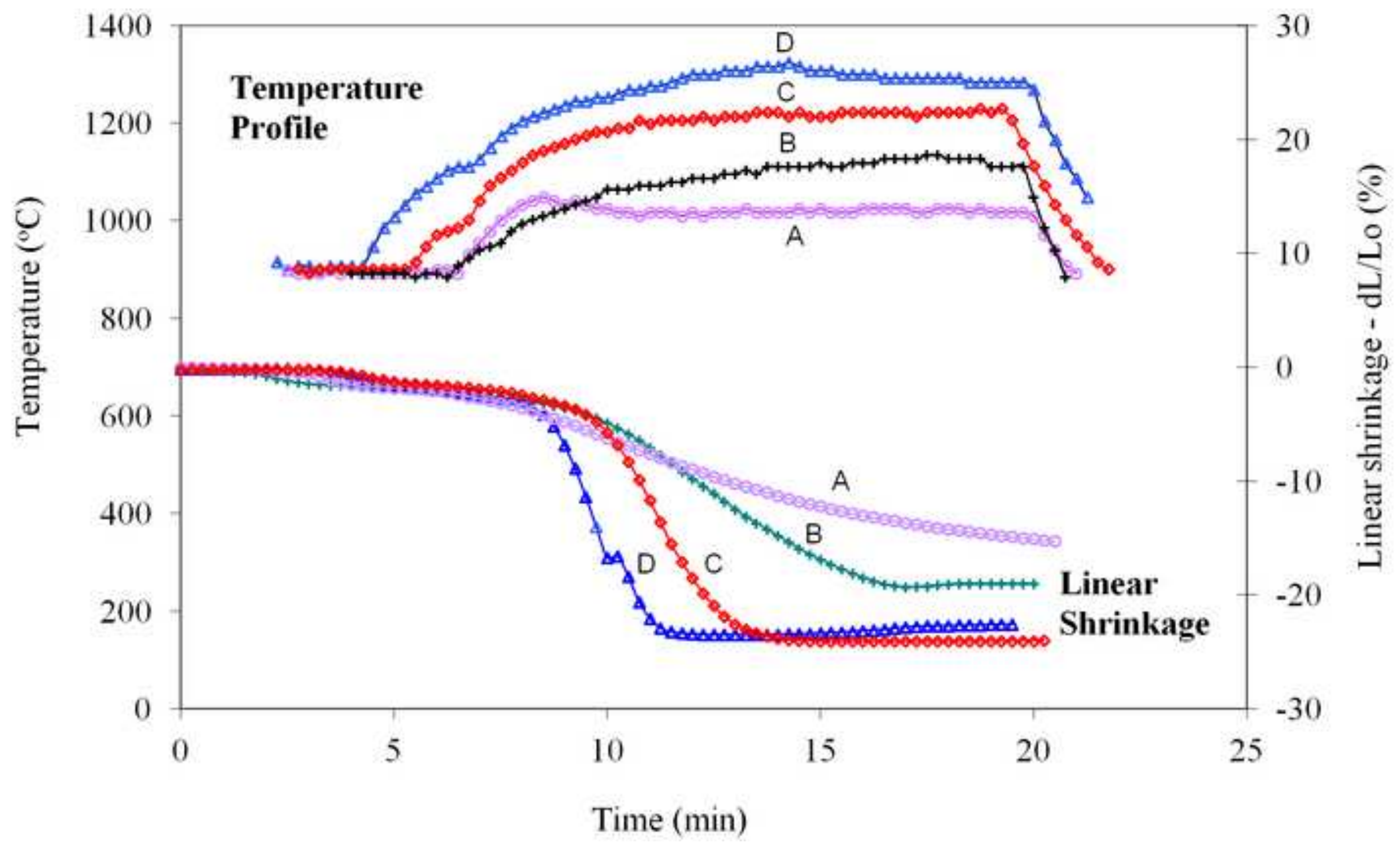




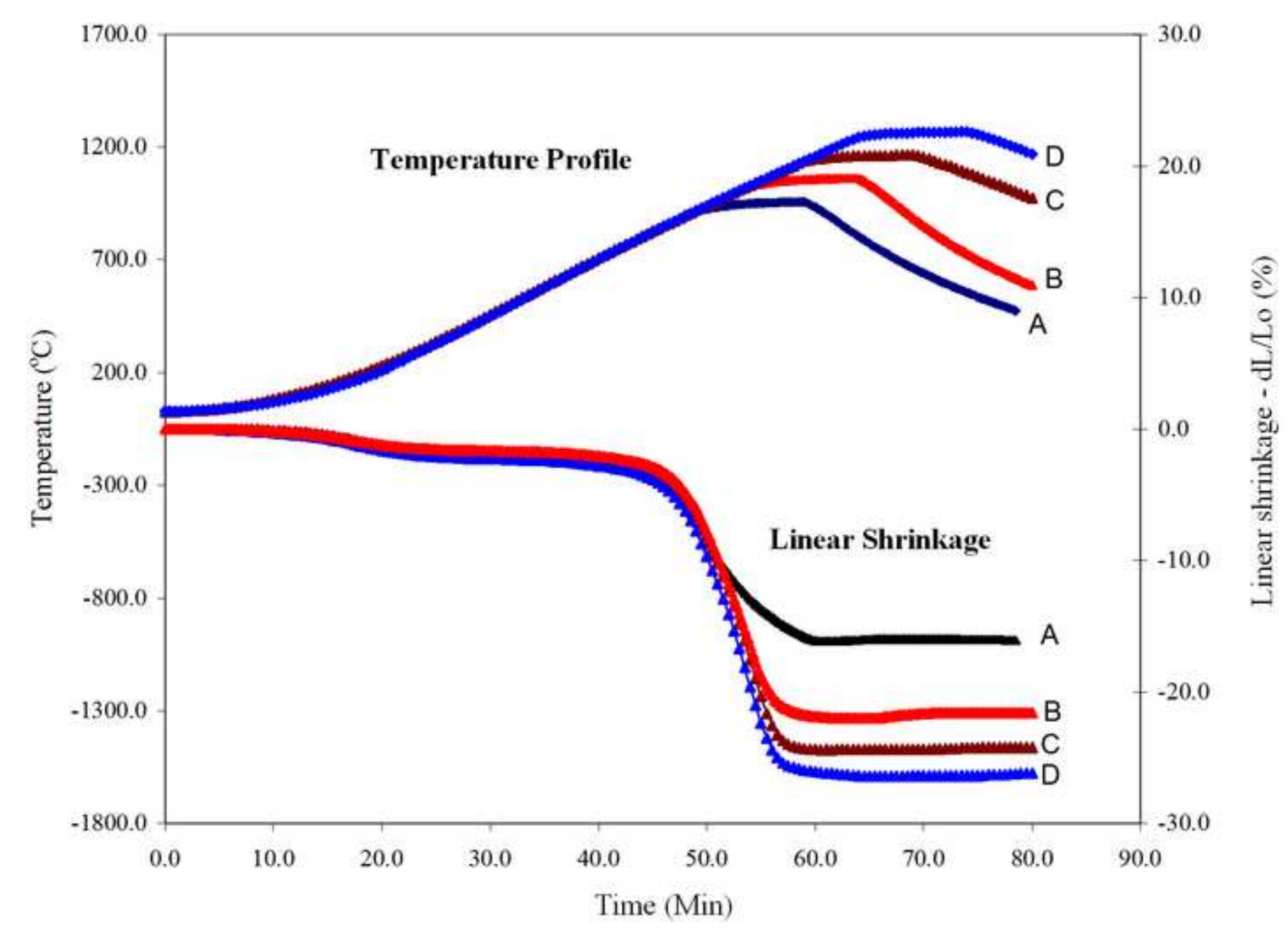

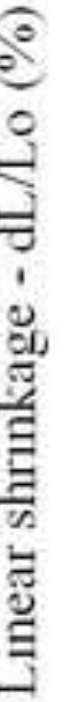

.




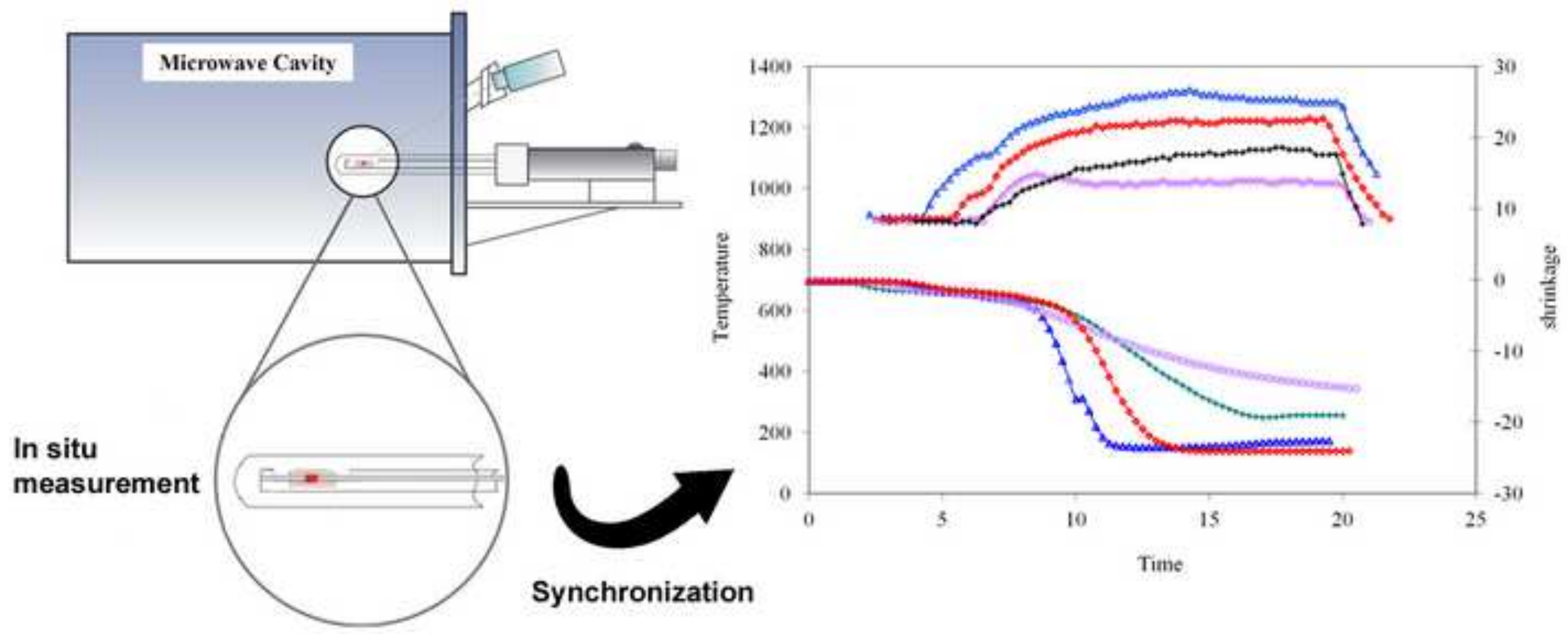

In situ measurement
Synchronization 